\title{
nutrients
}

ISSN 2072-6643

www.mdpi.com/journal/nutrients

Article

\section{Effect of Gliadin on Permeability of Intestinal Biopsy Explants from Celiac Disease Patients and Patients with Non-Celiac Gluten Sensitivity}

\author{
Justin Hollon 1,*, Elaine Leonard Puppa ${ }^{2}$, Bruce Greenwald ${ }^{3}$, Eric Goldberg ${ }^{3}$, \\ Anthony Guerrerio ${ }^{4}$ and Alessio Fasano ${ }^{5}$
}

1 Department of Pediatric Gastroenterology, Naval Medical Center Portsmouth, 620 John Paul Jones Circle, Portsmouth, VA 23708, USA

2 University of Maryland School of Medicine, Baltimore, MD 21201, USA;

E-Mail: eleonard@peds.umaryland.edu

3 Division of Gastroenterology and Hepatology, University of Maryland School of Medicine, Baltimore, MD 21201, USA; E-Mails: bgreenwa@medicine.umaryland.edu (B.G.); egoldber@medicine.umaryland.edu (E.G.)

4 Division of Pediatric Gastroenterology and Nutrition, Johns Hopkins University School of Medicine, Baltimore, MD 21205, USA; E-Mail: aguerrerio@jhmi.edu

5 Center for Celiac Research, Massachusetts General Hospital and Division of Pediatric Gastroenterology and Nutrition, Massachusetts General Hospital for Children, Boston, MA 02114, USA; E-Mail: afasano@partners.org

* Author to whom correspondence should be addressed; E-Mail: justin.hollon@med.navy.mil; Tel.: +757-953-4529; Fax: +757-953-3293.

Received: 28 October 2014 / Accepted: 11 February 2015 / Published: 27 February 2015

\begin{abstract}
Background: Intestinal exposure to gliadin leads to zonulin upregulation and consequent disassembly of intercellular tight junctions and increased intestinal permeability. We aimed to study response to gliadin exposure, in terms of barrier function and cytokine secretion, using intestinal biopsies obtained from four groups: celiac patients with active disease (ACD), celiac patients in remission (RCD), non-celiac patients with gluten sensitivity (GS) and non-celiac controls (NC). Methods: Ex-vivo human duodenal biopsies were mounted in microsnapwells and luminally incubated with either gliadin or media alone. Changes in transepithelial electrical resistance were monitored over $120 \mathrm{~min}$. Media was subsequently collected and cytokines quantified. Results: Intestinal explants from all groups $(\operatorname{ACD}(n=6), \operatorname{RCD}(n=6), \operatorname{GS}(n=6)$, and $\mathrm{NC}(n=5))$ demonstrated a greater increase in
\end{abstract}


permeability when exposed to gliadin $v s$. media alone. The increase in permeability in the ACD group was greater than in the RCD and NC groups. There was a greater increase in permeability in the GS group compared to the RCD group. There was no difference in permeability between the ACD and GS groups, between the RCD and NC groups, or between the NC and GS groups. IL-10 was significantly greater in the media of the NC group compared to the RCD and GS groups. Conclusions: Increased intestinal permeability after gliadin exposure occurs in all individuals. Following gliadin exposure, both patients with gluten sensitivity and those with active celiac disease demonstrate a greater increase in intestinal permeability than celiacs in disease remission. A higher concentration of IL-10 was measured in the media exposed to control explants compared to celiac disease in remission or gluten sensitivity.

Keywords: celiac disease; gluten sensitivity; IL-10

\section{Introduction}

Gluten is recognized as the environmental trigger of celiac disease (CD), an immune-mediated small intestinal enteropathy that has a prevalence in the United States of nearly 1\% [1]. In this condition, the reaction to gluten's immunogenic fraction, gliadin, is mediated by T-cell activation and is genetically associated with the human leukocyte antigen (HLA) alleles DQA1*0501/DQB1*0201. Diagnosis of CD is primarily reliant on histologic demonstration of the characteristic enteropathy, with supporting criteria, such as the presence of the celiac-specific antibodies to tissue transglutaminase (tTG) and HLA testing. Treatment of CD is lifelong maintenance of a gluten-free diet (GFD).

It is important to note that symptom alleviation on a GFD alone does not equate to a diagnosis of $\mathrm{CD}$ [2]. Along with the increasing awareness of CD has come the emerging recognition that there are individuals who clinically react to gluten-containing food ingestion without demonstration of a T-cell mediated process in the gastrointestinal mucosa. These individuals are classified as having non-celiac gluten sensitivity (GS) [3,4]. The rising awareness of GS is in part responsible for the growing popularity of the GFD, to the point that most people in the United States now on a GFD do not have CD [5]. While the symptoms of GS may mirror that of $\mathrm{CD}$, and these symptoms may resolve on a GFD, these individuals have no histologic evidence of small intestinal enteropathy while on a gluten-containing diet. Moreover, GS carries no association with an elevation of tTG autoantibodies, although several studies have shown a higher frequency of positive anti-gliadin antibodies (AGA) than the general population [6-8]. While the frequency of the HLA DQ2/8 genes is higher in GS than the general population, presence of these genes, unlike in CD, is not a prerequisite [6,8]. Generally established as a "diagnosis of exclusion", defining GS necessitates eliminating CD as the source of symptoms, followed by dietary elimination of gluten and, ultimately, reintroduction of gluten-containing foods in order to establish return of symptoms in association with gluten ingestion. This reintroduction is typically performed as an open challenge, although double-blind placebo controlled re-challenges have been performed $[9,10]$. 
The apparent lack of adaptive immune activation in GS raises the hypothesis that the similarities between GS and CD may be more related to a common defect in intestinal barrier function [11]. The intestinal barrier, by limiting uncontrolled access of non-self antigens in the lamina propria, is an integral part of immune surveillance, and impaired barrier function may play a role in a number of immune-mediated diseases, including CD [12-14]. In healthy intestinal epithelium, this intestinal barrier should be impermeable to macromolecules such as gliadin due to competent paracellular tight junctions. However, it has been well-demonstrated that antigenic gliadin peptides, which are inherently resistant to intraluminal digestion, are able to cross the intestinal epithelium of CD patients secondary to the gliadin-mediated, MyD88-dependent release of zonulin and consequent disassembly of the tight junction barrier $[15,16]$. In this study, we aimed to study whether gliadin causes a similar effect in non-celiac subjects by exposing, to gliadin, intestinal biopsy explants obtained from four different study groups: celiac patients with active disease (ACD), celiac patients in remission (RCD), non-celiac patients with gluten sensitivity (GS) and non-celiac controls (NC).

\section{Methods}

Biopsy specimens were taken from the second portion of the duodenum from adult subjects undergoing esophagogastroduodenoscopy (EGD) for clinically indicated reasons. All study subjects gave informed consent to undergo additional biopsies for the purpose of this study. This study was approved by the Institutional Review Board of the University of Maryland, Baltimore, and was conducted in accordance with their ethical standards and regulatory requirements.

\subsection{Study Group Categorization}

Clinically indicated biopsy specimens were submitted to pathology for standard reading by a pathologist with additional training in gastrointestinal pathology and were staged according to the Marsh Oberhuber classification [17]. Study subjects were classified into one of four different study groups: celiac patients with active disease (ACD, regular diet $\geq 2$ months, $n=6)$, celiac patients in remission (RCD, GFD $\geq 12$ months, $n=6$ ), non-celiac patients with gluten sensitivity (GS, regular diet $\geq 2$ months, $n=6$ ), and non-celiac controls (NC, regular diet, $n=5$ ).

For this study, all potential GS subjects were enrolled from the University of Maryland Center for Celiac Research in Baltimore, MD after presenting to clinic with a history of complete resolution of CD-like clinical symptoms after initiation of a GFD, yet no history of a previous EGD with duodenal biopsies while on a gluten-containing diet. To differentiate GS from CD, these patients were instructed to initiate an open (non-blinded) gluten challenge for a minimum of 2 months prior to endoscopy, with instruction to ingest a minimum of 10 grams of gluten daily, or the equivalent of 4 slices of wheat-based bread, and to subsequently proceed to endoscopy only if they experience a return of symptoms. The GS group was ultimately defined by those participants who had return of symptoms upon open gluten re-challenge and both negative CD serology (tTG and/or anti-endomysial antibodies (EMA)) and preserved duodenal villous architecture on histopathology (Marsh stage 0-1) [3]. Conversely, the ACD group was defined according to the modified 2012 criteria of the European Society of Pediatric Gastroenterology, Hepatology and Nutrition, as those participants who, after being on a gluten-containing 
diet for a minimum of 2 months, had biopsies demonstrating either villous blunting (Marsh 3) or, if accompanied by elevated tTG autoantibodies, crypt hyperplasia and intraepithelial lymphocytosis (Marsh 2) [18]. The RCD group was defined as having a previous biopsy-proven diagnosis of CD, with evidence of complete mucosal healing on the repeat biopsies taken at the time of the study (Marsh 0-1). These participants were maintained on a GFD prior to endoscopy. The NC group was defined as individuals that underwent an upper endoscopy because of dyspeptic symptoms; having demonstrated negative CD serology, Marsh 0-1 on duodenal histology, and no prior history of being on a GFD.

\subsection{Transepithelial Electrical Resistance (TEER) Measurements}

Intestinal permeability was assessed ex vivo by measuring transepithelial electrical resistance (TEER) of biopsy explants using a dual planar electrode (Endohm Evom; World Precision Instruments, Sarasota, FL), expressed in $\Omega \mathrm{cm}^{2}$ and normalized by the baseline resistance values. After their collection, four small-intestine biopsies were oriented on presterilized filter paper with the villi facing upward and mounted onto the modified microsnapwell system as previously described by El Asmar et al. [19]. After 30 min incubation at $37^{\circ} \mathrm{C}$, a baseline TEER measurement (time 0) was obtained. Subsequently, a pair of biopsy explants were then luminally exposed to Dulbecco's Modified Eagle's Medium (DMEM) alone and a pair of biopsy explants were exposed to pepsin-trypsin digested gliadin (PT-gliadin), prepared as previously described [20] and suspended in DMEM at a final concentration of $1 \mathrm{mg} / \mathrm{mL}$. TEER values were then monitored at $30 \mathrm{~min}$ intervals for a total of $120 \mathrm{~min}$. TEER values for each pair of biopsies were averaged and data was expressed as the fractional change from the baseline TEER.

\subsection{Cytokine Quantification}

Media was collected from the luminal (apical) and basolateral sides of the microsnapwell system at $120 \mathrm{~min}$ and cytokine levels were quantified using the Human ProInflammatory 7-Plex Ultra-Sensitive Kit (Meso Scale Discovery (MSD), Rockville, MD, US) which measures interleukin (IL)-12p70, IL-1 $\beta$, interferon (IFN)- $\gamma$, IL-6, IL-8, IL-10, and tumor necrosis factor (TNF)- $\alpha$ via multiplex electrochemiluminescence detection. MSD plates were analyzed on the MSD MS2400 imager. All standards and samples were measured in duplicate and the assays were performed according to the manufacturer's instructions. Cytokine levels were calculated using the manufacturer's software, given in $\mathrm{pg} / \mathrm{mL}$ and presented as medians with interquartile ranges. Per the manufacturer's instructions, the lower limit of detection, in $\mathrm{pg} / \mathrm{mL}$, for the assay is 0.77 for IL-12p70, 0.58 for IL- $1 \beta, 0.8$ for IFN- $\gamma, 0.18$ for IL-6, 0.10 for IL-8, 0.57 for IL-10, and 0.28 for TNF- $\alpha$.

\subsection{Statistical Analysis}

Differences within each group (between explants exposed to media alone and those exposed to PT-gliadin) were assessed by Wilcoxon matched pairs signed rank test; comparisons between groups at a given time point or condition were first assessed by a Kruskal-Wallis (KW) test. For time points showing significance by KW, Mann Whitney tests were performed on all pairs. For cytokine measurements, values and differences less than one half the lower limit of detection were set to one half the lower limit 
of detection. All data were analyzed and graphed using Prism version 6.0 software (GraphPad Software, La Jolla, CA, USA). A $p$ value $<0.05$ was considered statistically significant.

\section{Results}

Comparing within each group (Figure 1), there was a significant increase in permeability in intestinal explants exposed to PT-gliadin as compared to explants exposed to media alone for all time intervals $(30,60,90$ and $120 \mathrm{~min})$ in the ACD group ( $p=0.002,0.002,0.009,0.04$, respectively) and the GS group ( $p=0.02,0.009,0.02,0.004$, respectively). In the NC group, there was a significantly greater increase in permeability in the PT-gliadin exposed explants compared to those exposed to media alone at 30, 60 and $120 \mathrm{~min}$ ( $p=0.008,0.03,0.008$, respectively). In the RCD group, there was a significantly greater increase in permeability in the PT-gliadin exposed explants compared to those exposed to media alone at 60 min only $(p=0.04)$.
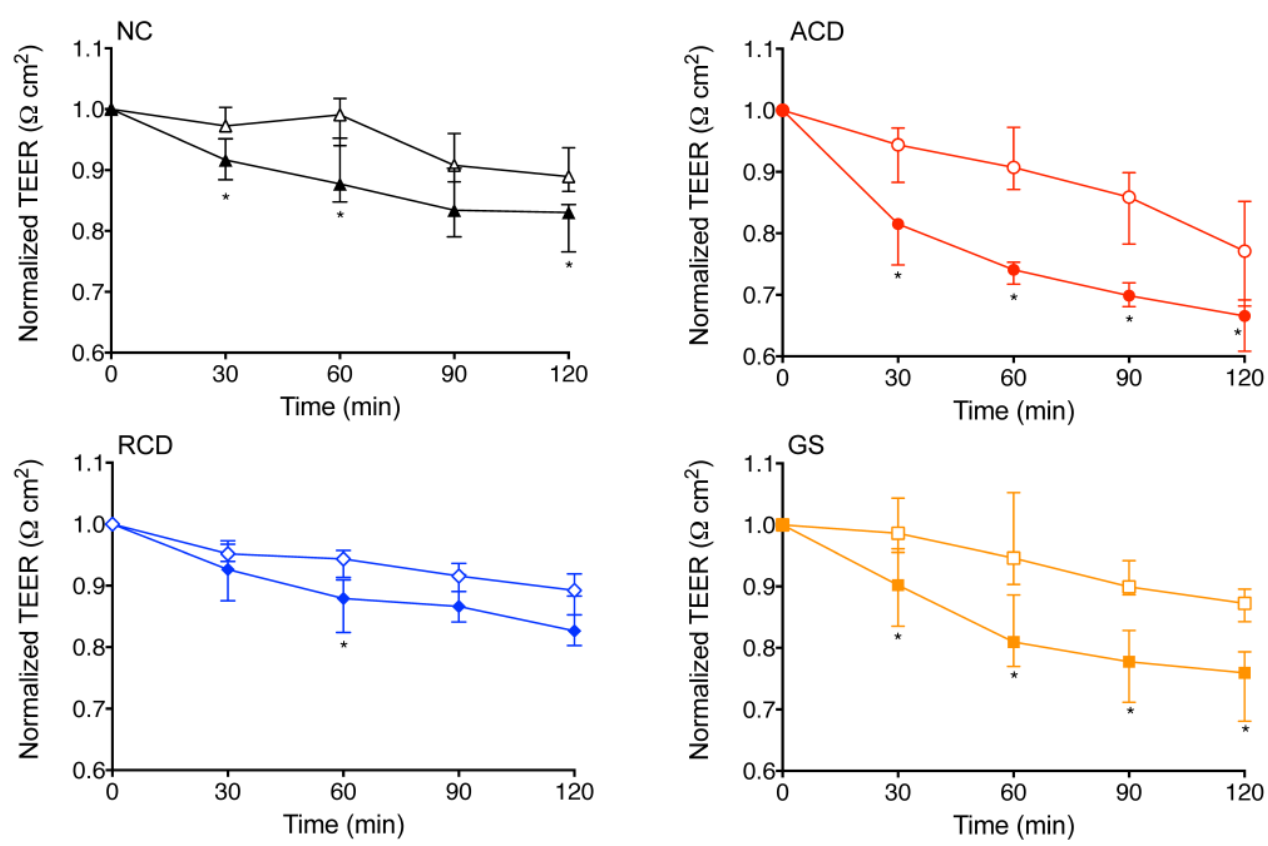

Figure 1. Normalized transepithelial electrical resistance (TEER) changes in human intestinal explants exposed to PT-gliadin or media alone. Explants obtained from non-celiac controls (NC), celiac patients with active disease (ACD), celiac patients in remission (RCD), and non-celiac patients with gluten sensitivity (GS). Explants were exposed to media alone (open symbols) or to PT-gliadin (filled symbols). NC (black triangle) explants demonstrated a greater increase in permeability (decrease in TEER) when exposed to PT-gliadin vs. exposure to media alone at 30,60 and $120 \mathrm{~min}$. RCD (blue diamond) explants demonstrated a greater increase in permeability when exposed to PT-gliadin vs exposure to media alone at 60 and 120 min. ACD (red circle) and GS (gold square) explants demonstrated a greater increase in permeability when exposed to PT-gliadin vs exposure to media alone at 30, 60, 90 and 120 min. Symbol denotes the median. Whiskers denote the 25 th to 75 th percentile. ${ }^{*} p<0.05$. 
When comparing between groups, intestinal explants incubated in media alone showed no significant difference in permeability except at $30 \mathrm{~min}$ between the RCD and NC groups ( $p=0.03$ ) (Figure 2A). In order to assess for the isolated effect of exposure to PT-gliadin at each measurement interval, exposures to media alone in each study group were used as the baseline TEER values that were subtracted from the corresponding values of the PT-gliadin exposed tissue explants (Figure 2B). As shown in Figure 2B, following gliadin exposure, the increase in permeability over baseline in explants from the ACD group was significantly greater than that of the explants from the RCD group at 30, 60 and $90 \mathrm{~min}(p=0.004$, $0.004,0.02$, respectively). The GS group had a significant increase in permeability over baseline compared to the RCD group at $90 \mathrm{~min}(p=0.03)$. Increase in permeability over baseline in explants from the ACD group was significantly greater than that of the explants from the NC group at $30 \mathrm{~min}$ $(p=0.02)$. There was no significant difference in permeability between explants from the RCD and NC groups, the NC and GS groups, or the GS and ACD groups. There was no significant difference in permeability between explants from the RCD and NC groups, the NC and GS groups, or the GS and ACD groups. Coefficient of variation (mean \pm standard deviation) for the TEER measurements were $16 \% \pm 20 \%, 8.5 \% \pm 6.5 \%, 2.8 \% \pm 1.3 \%$, and $6.4 \% \pm 5.6 \%$ for the GS, ACD, RCD, and NC samples, respectively, in media alone, while it was $11 \% \pm 10 \%, 17 \% \pm 14 \%, 5.8 \% \pm 3.6 \%$, and $5.1 \% \pm 3.2 \%$, respectively, in the media plus PT-gliadin samples.

After $2 \mathrm{~h}$, production of the anti-inflammatory cytokine IL-10 was significantly greater for the NC group compared to the GS and RCD groups in media alone $(p=0.01,0.03)$ and in PT-gliadin containing media collected from the basolateral side of explants $(p=0.01,0.03)$ (Figure 3 ). There was no significant difference in the change of measured levels of the proinflammatory cytokines IL-12p 70 , IL-1 $\beta$, IFN- $\gamma$, IL-6, IL-8, or TNF- $\alpha$ on exposure to PT-gliadin in either the luminally or basolaterally exposed media and in IL-10 in the luminally exposed media (data not shown).

\section{Discussion}

$\mathrm{CD}$ is mediated by T-cell activation against gluten's immunogenic fraction, gliadin; specifically, a 33-mer peptide fragment that is resistant to intraluminal proteolysis and serves as the primary antigen for T-cell proliferation [21]. Activation of the adaptive immune response requires specific HLA class II genes and is typically accompanied by specific celiac-associated IgA antibodies; the end result of this inflammatory cascade - the characteristic enteropathy - must be histologically demonstrated for a diagnosis of CD to be made. However, there is an emerging recognition that there are individuals who react to gluten-containing food in a manner symptomatically indistinguishable from CD yet without the typical CD serology or associated histopathology. These individuals are defined as having non-celiac gluten sensitivity (GS) $[3,4]$. Adverse reactions to gluten outside the gastrointestinal tract, such as dermatitis herpetiformis and gluten ataxia [22], have been well-studied, yet research has only recently begun to clearly define the spectrum of gastrointestinal gluten-related disorders, and there are no objective laboratory markers to-date that are specific for GS. As the absence of an adaptive immune response in GS distinguishes this condition from $\mathrm{CD}$, the aim of this study was to further evaluate the role of the intestinal barrier in these two conditions, both in terms of its barrier function and cytokine secretion. 

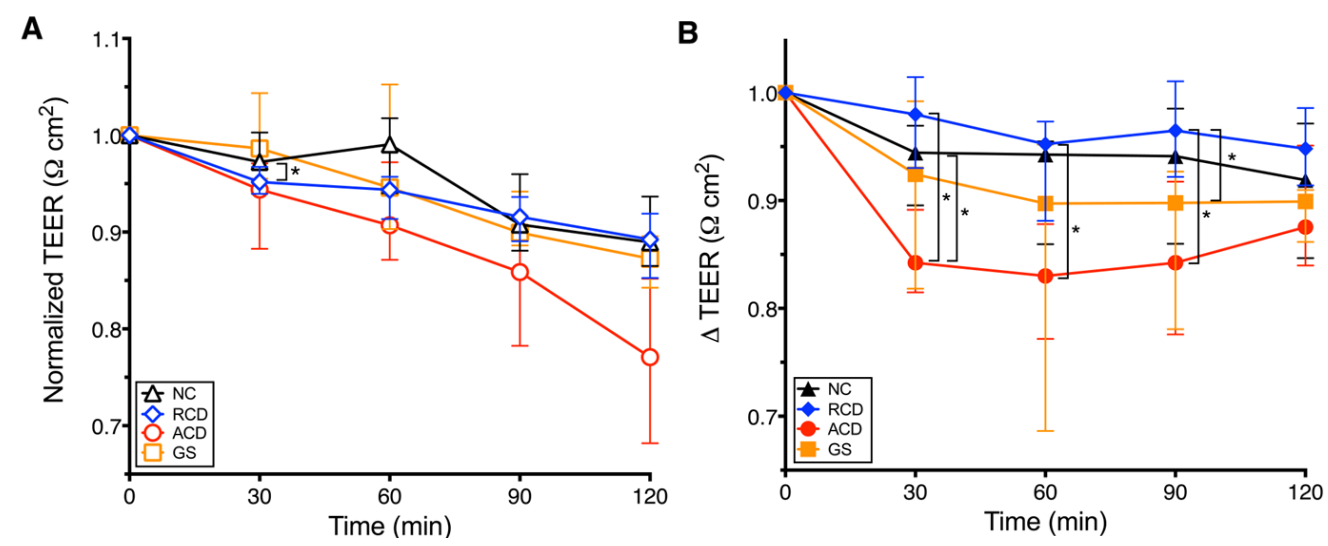

Figure 2. Comparison between groups of gut permeability changes induced by either PT-gliadin or media alone measured as normalized transepithelial electrical resistance changes ( $\triangle$ TEER) in human intestinal explants. Explants obtained from celiac patients in remission (RCD), non-celiac controls (NC), non-celiac patients with gluten sensitivity (GS), and celiac patients with active disease (ACD). A decrease in TEER indicates increased gut permeability. A. TEER changes of explants exposed to media alone. Explants incubated in media alone demonstrated no significant differences in permeability except between NC (black triangle) and RCD (blue diamond) groups at $30 \mathrm{~min}$. B. TEER of PT-gliadin exposed explants minus TEER of corresponding explants exposed to media alone ( $\triangle$ TEER). Increases in permeability in explants from the ACD group (red circle) were significantly greater than in explants from the RCD group (blue diamond) at 30,60 and 90 min, and the NC group (black triangle) at $30 \mathrm{~min}$. Increases in permeability in explants from the GS group (gold square) were significantly greater than in explants from the RCD group at $90 \mathrm{~min}$. There was no significant difference in permeability between explants from the ACD and GS groups, between explants from the RCD and NC groups, or between explants from the NC and GS groups. Symbol denotes the median. Whiskers denote the 25 th to 75 th percentile. ${ }^{*} p<0.05$.

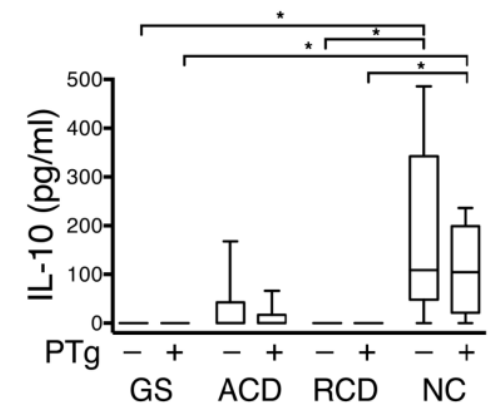

Figure 3. Comparison between groups of IL-10 secretion from the basolateral side of biopsy explants after $120 \mathrm{~min}$, with and without PT-gliadin exposure. Media collected from the basolateral sides of explants obtained from non-celiac patients with gluten sensitivity (GS), celiac patients with active disease (ACD), celiac patients in remission (RCD), and non-celiac controls (NC). The NC group showed concentrations of IL-10 significantly higher than the corresponding media from the GS and RCD group. In the graphs, the box defines the 25 th and 75 th percentile; center line the median; whiskers the range. ${ }^{*} p<0.05$. 
Due to the integrity of intestinal tight junctions, normal intestinal epithelium should be impermeable to such indigestible macromolecules such as the 33-mer gliadin peptide fragment, yet in CD these antigens are able to pass from the lumen to the lamina propria. Compromised permeability to macromolecules appears to be independent of the enteropathy encountered in $\mathrm{CD}$, with research demonstrating that unaffected first-degree relatives of CD patients similarly have increased intestinal permeability at baseline [23]. The mechanisms of gliadin-induced permeability in CD have been well-studied and include the interaction of specific gliadin peptides with the CXCR3 receptor expressed on the luminal side of enterocytes, paracellular trafficking via zonulin release, and subsequent tight junction disassembly [15,16,24,25]. Enhanced intestinal permeability upon gliadin exposure does not appear to be limited to CD patients. A previous ex vivo study conducted by Drago et al. using duodenal biopsy explants in a microsnapwell system demonstrated that, following $60 \mathrm{~min}$ of PT-gliadin exposure, tissue from both RCD patients and controls demonstrated a significant increase in intestinal permeability as well as a temporally-associated increase in zonulin release [20]. Moreover, a recently published study by Vazquez-Roque et al. using lactulose/mannitol (LA/MA) permeability testing in patients with diarrhea-predominant irritable bowel syndrome (IBS) demonstrated higher small bowel permeability in patients randomized to a 4-week gluten-containing diet compared to those randomized to a four-week GFD [26].

In order to investigate the role of gut barrier dysfunction in gluten-related disorders, a study conducted by Sapone et al. performed LA/MA permeability testing on GS, ACD and dyspeptic (control) patients [8]. The ACD group demonstrated a significantly higher urinary LA/MA ratio (increased intestinal permeability) than the GS group. However there was not an associated increase in the urinary LA/MA ratio when compared to the controls in either the ACD or the GS population. This urinary LA/MA ratio was performed on patients after a period of fasting and, as such, is a marker of baseline permeability. In our ex vivo study, this is akin to the baseline, or media-only, TEER study arm shown in Figure 2A. Like the Sapone et al. study, our TEER results of the media-only biopsy explants demonstrated no increase in permeability in the GS group compared to the NC group, or in the ACD group compared to the NC group.

Figures 1 and 2B illustrate the effects on intestinal permeability after direct exposure to PT-gliadin. Similar to the previously mentioned study on RCD and control patients conducted by Drago et al. we found a statistically significant increase in intestinal permeability upon PT-gliadin exposure across all four study groups (Figure 1). Figure 2B demonstrates that, when comparing between groups, upon PT-gliadin exposure, the GS explants reacted similarly to the ACD explants, with no statistical difference between these groups. The NC explants reacted similarly to the RCD explants, likewise with no statistical difference between these groups. Both the ACD and GS explants had a statistically greater increase in permeability than the RCD explants, and explants from the NC and RCD groups reacted to a lesser degree than those from the ACD group. These results complement those previously reported by Sapone et al.; although GS patients have a normal intestinal barrier at baseline, gliadin exposure induces increased intestinal permeability in these patients, with a response that closely resembles that observed in the ACD group and is more pronounced than that observed in the RCD group.

The RCD group demonstrated the smallest degree in permeability change upon exposure to PT-gliadin and the difference reached significance at only $60 \mathrm{~min}$. This is a delay when compared to the other study groups, which all demonstrated an increase in permeability at the first measured interval of 
$30 \mathrm{~min}$. The RCD group was the only group in our study on a strict GFD prior to endoscopy, suggesting that the gluten-induced activation of the zonulin pathway is comparatively delayed in intestinal tissue that is not routinely exposed to dietary gluten, even in those patients with celiac disease. Similarly, the randomized controlled trial by Vazquez-roque et al. demonstrated significantly increased small bowel permeability by (LA/MA) testing in IBS patients randomized to a gluten-containing diet compared to those randomized to 4 weeks of a strict GFD [26]. Given that symptom-onset upon gluten-containing food exposure in the GS population is comparatively faster than in patients with $\mathrm{CD}$, further permeability experiments comparing GS patients on a strict GFD to those with RCD would help contribute to the understanding of the timing of these alterations.

Cytokine quantification from the media collected from the luminal and basolateral sides of the biopsy explants after $120 \mathrm{~min}$ of PT-gliadin exposure failed to show any significant difference in the classic inflammatory cytokines, to include IL-6, IL-8, IFN- $\gamma$, and TNF- $\alpha$. The relatively short period of incubation may in part explain the lack of detection of an inflammatory innate response to gluten exposure. Production of the anti-inflammatory cytokine IL-10 was significantly higher in the media collected from the basolateral side of the explants obtained from the NC group as compared to the GS and RCD groups (Figure 3) in both the unexposed media and the media exposed to PT-gliadin. IL-10 is a key mediator for regulating the innate intestinal immune response [27]. A study by Madsen et al. in IL-10 deficient mice demonstrated that IL-10 plays a significant role in intestinal permeability and, in fact, deficiencies of IL-10 lead to increased intestinal permeability prior to the development of mucosal inflammation [28]. We do not suggest that patients with GS or CD are IL-10 deficient; however, this degree of IL-10 production in the NC group may be indicative of a more competent innate immune response. Further studies will be needed to help elucidate if this lack of secretion of IL-10 is a primary factor leading to an exaggerated increase in PT-gliadin induced permeability or a secondary marker of a separate defect in innate immunity.

A primary limitation of this study relates to the lack of objective laboratory biomarkers specific for GS. Currently, the diagnosis is based on exclusion criteria; specifically these patients should have negative CD serology (anti-EMA and/or anti-tTG), normal duodenal histopathology, and resolution of CD-like symptoms on a GFD with subsequent return of symptoms upon dietary reintroduction of gluten-containing food [3]. For the purposes of this study, our GS patients had undergone an open gluten challenge and we recognize that such a method could allow for the possibility of patients without true GS being included in the GS group due to placebo-response. While dietary re-challenge in a blinded fashion would have helped avoid the possibility of a placebo effect, the necessity of a gluten-containing diet at biopsy precluded this type of intervention. Similarly, no patients in our control group had previously attempted a GFD; ideally, the control group would have been comprised of those who had already had GS ruled-out by a negative GFD trial. The percentage of patients with IBS who truly have GS may be as high as 30\%; given that the indication for duodenal biopsies in our NC group would include the non-specific gastrointestinal symptoms of CD and GS, it is conceivable that some of the NC patients may have undiagnosed GS [9]. 


\section{Conclusions}

This study demonstrates that gliadin exposure induces an increase in intestinal permeability in all individuals, regardless of whether or not they have celiac disease. The results of this study suggest that gluten exposure leads to altered barrier function in both ACD and GS, resulting in an exaggerated increase in intestinal permeability when compared to RCD. The intestinal mucosal secretion of IL-10 from the basolateral surface seen in NC subjects in this study was not observed in those with RCD or GS. Specific laboratory markers for GS are still necessary to allow for a more objective definition of GS and further research into GS disorders would benefit from double-blind, placebo-controlled studies.

\section{Acknowledgments}

The authors thank Karen Lammers, Rosaria Fiorentino and Stefania Senger (Center for Celiac Research, Boston, MA, USA) for their technical help and expertise. The authors are grateful to Carmen Cuffari (Johns Hopkins Pediatric Gastroenterology Fellowship Program) for his guidance and encouragement. The views expressed in this article are those of the authors and do not necessarily reflect the official policy or position of the United States Department of Defense.

\section{Author Contributions}

The authors' responsibilities were as follows-A.F. conceived and designed the study. J.H., E.L. and A.F. contributed to the design of the study and participated in subject recruitment. J.H. conducted the study and was the primary author for the manuscript. B.G. and E.G. participated in the recruitment of the patients and carried out the endoscopy procedures. J.H., T.G. and A.F. were involved in the analysis and interpretation of the data. T.G. and A.F. critically revised the manuscript. All authors approved the final version of the manuscript.

\section{Conflicts of Interest}

The authors declare no conflict of interest.

\section{References}

1. Fasano, A.; Berti, I.; Gerarduzzi, T.; Not, T.; Colletti, R.B.; Drago, S.; Elitsur, Y.; Green, P.H.; Guandalini, S.; Hill, I.D.; et al. Prevalence of celiac disease in at-risk and not-at-risk groups in the United States: A large multicenter study. Arch. Intern. Med. 2003, 163, 286-292.

2. Campanella, J.; Biagi, F.; Bianchi, P.I.; Zanellati, G.; Marchese, A.; Corazza, G.R. Clinical response to gluten withdrawal is not an indicator of coeliac disease. Scand. J. Gastroenterol. 2008, 43, 1311-1314.

3. Sapone, A.; Bai, J.C.; Ciacci, C.; Dolinsek, J.; Green, P.H.R.; Hadjivassiliou, M.; Kaukinen, K.; Rostami, K.; Sanders, D.S.; Schumann, M.; et al. Spectrum of gluten-related disorders: Consensus on new nomenclature and classification. BMC Med. 2012, 10, 13, doi:10.1186/1741-7015-10-13. 
4. Ludvigsson, J.F.; Leffler, D.A.; Bai, J.C.; Biagi, F.; Fasano, A.; Green, P.H.; Hadjivassiliou, M.; Kaukinen, K.; Kelly, C.P.; Leonard, J.N.; et al. The Oslo definitions for coeliac disease and related terms. Gut 2012, 62, 43-52.

5. Rubio-Tapia, A.; Ludvigsson, J.F.; Brantner, T.L.; Murray, J.A.; Everhart, J.E. The prevalence of celiac disease in the United States. Am. J. Gastroenterol. 2012, 107, 1538-1544.

6. Wahnschaffe, U.; Schulzke, J.D.; Zeitz, M.; Ullrich, R. Predictors of clinical response to gluten-free diet in patients diagnosed with diarrhea-predominant irritable bowel syndrome. Clin. Gastroenterol. Hepatol. 2007, 5, 844-850.

7. Volta, U.; Tovoli, F.; Cicola, R.; Parisi, C.; Fabbri, A.; Piscaglia, M.; Fiorini, E.; Caio, G. Serological tests in gluten sensitivity (nonceliac gluten intolerance). J. Clin. Gastroenterol. 2012, 46, 680-685.

8. Sapone, A.; Lammers, K.M.; Casolaro, V.; Cammarota, M.; Giuliano, M.T.; de Rosa, M.; Stefanile, R.; Mazzarella, G.; Tolone, C.; Russo, M.I.; et al. Divergence of gut permeability and mucosal immune gene expression in two gluten-associated conditions: Celiac disease and gluten sensitivity. BMC Med. 2011, 9, 23, doi:10.1186/1741-7015-9-23.

9. Carroccio, A.; Mansueto, P.; Iacono, G.; Soresi, M.; D’Alcamo, A.; Cavataio, F.; Brusca, I.; Florena, A.M.; Ambrosiano, G.; Seidita, A.; et al. Non-celiac wheat sensitivity diagnosed by double-blind placebo-controlled challenge: Exploring a new clinical entity. Am. J. Gastroenterol. 2012, 107, 1898-1906.

10. Biesiekierski, J.R.; Newnham, E.D.; Irving, P.M.; Barrett, J.S.; Haines, M.; Doecke, J.D.; Shepherd, S.J.; Muir, J.G.; Gibson, P.R. Gluten causes gastrointestinal symptoms in subjects without celiac disease: A double-blind randomized placebo-controlled trial. Am. J. Gastroenterol. 2011, 106, 508-514.

11. Verdu, E.F.; Armstrong, D.; Murray, J.A. Between celiac disease and irritable bowel syndrome: The "no man's land" of gluten sensitivity. Am. J. Gastroenterol. 2009, 104, 1587-1594.

12. Liu, Z.; Li, N.; Neu, J. Tight junctions, leaky intestines, and pediatric diseases. Acta Paediatr. 2005, 94, 386-393.

13. Monsuur, A.J.; Wijmenga, C. Understanding the molecular basis of celiac disease: What genetic studies reveal. Ann. Med. 2006, 38, 578-591.

14. Fasano, A.; Shea-Donohue, T. Mechanisms of disease: The role of intestinal barrier function in the pathogenesis of gastrointestinal autoimmune diseases. Nat. Clin. Pract. Gastroenterol. Hepatol. 2005, 2, 416-422.

15. Fasano, A.; Not, T.; Wang, W.; Uzzau, S.; Berti, I.; Tommasini, A.; Goldblum, S.E. Zonulin, a newly discovered modulator of intestinal permeability, and its expression in coeliac disease. Lancet $\mathbf{2 0 0 0}$, 355, 1518-1519.

16. Cummins, A.G.; Thompson, F.M.; Butler, R.N.; Cassidy, J.C.; Gillis, D.; Lorenzetti, M.; Southcott, E.K.; Wilson, P.C. Improvement in intestinal permeability precedes morphometric recovery of the small intestine in coeliac disease. Clin. Sci. (Lond.) 2001, 100, 379-386.

17. Marsh, M.N. The natural history of gluten sensitivity: Defining, refining and re-defining. QJM 1995, 88, 9-13. 
18. Husby, S.; Koletzko, S.; Korponay-Szabo, I.R.; Mearin, M.L.; Phillips, A.; Shamir, R.; Troncone, R.; Giersiepen, K.; Branski, D.; Catassi, C.; et al. European society for pediatric gastroenterology, hepatology, and nutrition guidelines for the diagnosis of coeliac disease. $J$. Pediatr. Gastroenterol. Nutr. 2012, 54, 136-160.

19. El Asmar, R.; Panigrahi, P.; Bamford, P.; Berti, I.; Not, T.; Coppa, G.V.; Catassi, C.; Fasano, A. Host-dependent zonulin secretion causes the impairment of the small intestine barrier function after bacterial exposure. Gastroenterology 2002, 123, 1607-1615.

20. Drago, S.; El Asmar, R.; Di Pierro, M.; Grazia Clemente, M.; Tripathi, A.; Sapone, A.; Thakar, M.; Iacono, G.; Carroccio, A.; D'Agate, C.; et al. Gliadin, zonulin and gut permeability: Effects on celiac and non-celiac intestinal mucosa and intestinal cell lines. Scand. J. Gastroenterol. 2006, 41, 408-419.

21. Shan, L.; Molberg, O.; Parrot, I.; Hausch, F.; Filiz, F.; Gray, G.M.; Sollid, L.M.; Khosla, C. Structural basis for gluten intolerance in celiac sprue. Science 2002, 297, 2275-2279.

22. Hadjivassiliou, M.; Sanders, D.S.; Grunewald, R.A.; Woodroofe, N.; Boscolo, S.; Aeschlimann, D. Gluten sensitivity: From gut to brain. Lancet Neurol. 2010, 9, 318-330.

23. Van Elburg, R.M.; Uil, J.J.; Mulder, C.J.; Heymans, H.S. Intestinal permeability in patients with coeliac disease and relatives of patients with coeliac disease. Gut 1993, 34, 354-357.

24. Tripathi, A.; Lammers, K.M. Goldblum, S.; Shea-Donohue, T.; Netzel-Arnett, S.; Buzza, M.S.; Antalis, T.M.; Vogel, S.N.; Zhao, A.; Yang, S.; et al. Identification of human zonulin, a physiological modulator of tight junctions, as prehaptoglobin-2. Proc. Natl. Acad. Sci. USA 2009, 106, 16799-16804.

25. Lammers, K.M.; Lu, R.; Brownley, J.; Lu, B.; Gerard, C.; Thomas, K.; Rallabhandi, P.; Shea-Donohue, T.; Tamiz, A.; Alkan, S.; et al. Gliadin induces an increase in intestinal permeability and zonulin release by binding to the chemokine receptor CXCR3. Gastroenterology 2008, 135, 194-204.e3.

26. Vazquez-Roque, M.I.; Camilleri, M.; Smyrk, T.; Murray, J.A.; Marietta, E.; O’Neill, J.; Carlson, P.; Lamsam, J.; Janzow, D.; Eckert, D.; et al. A controlled trial of gluten-free diet in patients with irritable bowel syndrome-diarrhea: Effects on bowel frequency and intestinal function. Gastroenterology 2013, 144, 903-911.e3.

27. Moore, K.W.; de Waal Malefyt, R.; Coffman, R.L.; O'Garra, A. Interleukin-10 and the interleukin10 receptor. Annu. Rev. Immunol. 2001, 19, 683-765.

28. Madsen, K.L.; Malfair, D.; Gray, D.; Doyle, J.S.; Jewell, L.D.; Fedorak, R.N. Interleukin-10 gene-deficient mice develop a primary intestinal permeability defect in response to enteric microflora. Inflamm. Bowel Dis. 1999, 5, 262-270.

(C) 2015 by the authors; licensee MDPI, Basel, Switzerland. This article is an open access article distributed under the terms and conditions of the Creative Commons Attribution license (http://creativecommons.org/licenses/by/4.0/). 